\title{
The rod-and-frame illusion in erect and supine observers
}

\author{
DONALD R. GOODENOUGH, PHILIP K. OLTMAN, ERIC SIGMAN, \\ and PATRICIA W. COX \\ Educational Testing Service, Princeton, New Jersey 08541
}

\begin{abstract}
Previous studies have found that the effect of a tilted frame on egocentric rod adjustments is greater when an overhead display in a horizontal plane is viewed from a supine body position than when a vertical display is viewed from an erect body position. The present studies were designed to see whether this phenomenon could be attributed to an intravisual orientation contrast effect or to the effects of visually induced eye torsion. No significant erect-supine differences were found on measures of either effect. Errors in the direction of frame tilt were significantly greater in the supine position when observers were asked to align a visible rod or an unseen hand-held disk with the head, but no effect of body position was found in matching the orientation of the disk with the rod. The data suggest that erect-supine differences in frame effects are not attributable simply to intravisual factors. The results are discussed in terms used by Harris (1974) to describe "straight-ahead shifts" in judging spatial directions with respect to the median plane.
\end{abstract}

When an erect observer views a vertically or horizontally oriented rod that is surrounded by a large, laterally tilted frame, the rod looks tilted under most conditions. In the range of frame tilts between 0 and $45 \mathrm{deg}$, the rod looks tilted in the direction opposite to the frame. This rod-and-frame (RF) illusion can be measured in several ways. Most commonly, the observer is asked to adjust the rod to the apparent gravitational vertical. Rod settings under these conditions tend to err in the direction of frame tilt (e.g., Lichtenstein \& Saucer, 1974; Witkin, Dyk, Faterson, Goodenough, \& Karp, 1962/1974). Similar, but somewhat smaller, errors have usually been found with egocentric instructions to align the rod with an axis of the body (e.g., Brosgole \& Cristal, 1967; Sigman, Goodenough, \& Flannagan, 1979). The RF illusion has also been measured by kinesthetic matching methods in which the observer is asked to align an unseen, hand-held indicator with a vertical or horizontal rod. Kinesthetic settings tend to err in the direction of the illusory tilt of the rod (e.g., Gogel \& Newton, 1975; Goodenough, Oltman, Sigman, Rosso, \& Mertz, 1979).

The egocentric rod-adjustment method has been used in several studies to examine the effects of body position on the RF illusion (Brosgole \& Cristal, 1967; Templeton, 1973). The findings suggest that the illusory effect of the tilted frame is larger when an overhead display in a horizontal plane is viewed from the supine position than when a display in a vertical plane is viewed from the erect position. Since the gravitational direction is irrelevant to the definition

This work was conducted with the aid of NIMH Grant MH-21989. of rod tilt in a horizontal plane, this result suggests that gravitational cues aid in egocentric rod adjustments in the usual erect position (e.g., Templeton, 1973). It is not clear why this should be so, however.

Adjustments of the rod to an axis of the head require information about the orientation of the rod with respect to the axes of the retina and about the orientation of the retina with respect to the axes of the head. There is evidence to suggest that egocentric rod-adjustment errors may be due to distortions induced by the tilted frame in each type of information.

Information about retinal orientation with respect to the head may be distorted by eye torsion (ocular rotation around the line of sight). Visually induced torsion in the direction of display tilt has been found in several studies (e.g., Crone, 1975; Goodenough, Sigman, Oltman, Rosso, \& Mertz, 1979; Hughes, 1973). When such torsion occurs, the image of the rod is rotated in the opposite direction on the retina. Visually induced eye torsion is not very great (less than $1 \mathrm{deg}$ ), but individual differences in amount of torsion are related to individual differences in rodadjustment errors, as would be expected if the illusory effect of the frame is partly due to perceptually uncompensated eye torsion (Hughes, 1973). Thus, a rod that is objectively aligned with an axis of the head may look tilted away from that axis because of an incorrect information processing assumption that the axes of head and eye coincide.

Information about rod orientation with respect to the retinal axes may be distorted by an intravisual orientation contrast effect. There is a variety of evidence that the presence of a visual line at one orientation affects the apparent tilt of a second line at a different orientation (e.g., Carpenter \& Blakemore, 
1973). For orientation differences in the $10-$ to $30-\mathrm{deg}$ range, at least, the lines look more tilted away from each other than they actually are. It has been suggested that this contrast effect may be due to inhibitory interactions among orientation-selective neural units in the visual cortex (Carpenter \& Blakemore, 1973). There is some evidence suggesting that orientation contrast effects of this sort occur with the rod-and-frame display under some conditions (Gogel \& Newton, 1975; Goodenough, Oltman et al., 1979). Thus, a rod that is objectively aligned with the normally vertical retinal meridian may look tilted away from the frame because of the contrast in orientation between the rod and the frame sides that are most nearly parallel to the rod.

One question that remains to be answered is whether frame effects on egocentric rod adjustments can be fully attributed to a combination of torsion and contrast components. It has been difficult to answer this question directly because of problems in measuring the separate components under the same conditions (Goodenough, Oltman et al., 1979). There is reason to doubt, however, that an increase in the egocentric RF illusion in the supine position could be explained by changes in either torsion or contrast effects. An erect-supine difference in the contrast component seems unlikely, particularly since similar contrast effects are evidently locked to retinal rather than gravitational coordinates when body position is varied by lateral tilting (e.g., Wallace \& Moulden, 1973). The effects of a stationary tilted frame on eye torsion have not been studied in supine observers, but no erect-supine difference has been found in torsional responses to a rotating visual display (Finke \& Held, 1978). If the advantage of the erect body position cannot be attributed to either contrast or torsion effects, then the conclusion would seem warranted that some as-yet unspecified additional component contributes to the egocentric RF illusion. The experiments described below were designed to examine this question.

\section{EXPERIMENT 1}

In the attempt to replicate previous findings on the effect of body position, 20 young adult females were asked to make erect and supine egocentric rod adjustments in the absence and presence of a frame tilted by $22.5 \mathrm{deg}$. The subjects' task was to set the rod orthogonal to the longitudinal axis of the head (i.e., parallel to an imaginary line running through the center of both eyes). Frame orientations and rod settings are described as 0 deg if parallel to this reference line. Clockwise (CW) deviations from the 0 -deg reference are assigned a positive value, and counterclockwise (CCW) deviations are assigned a negative value.

The RF illusion was also measured, under the frame and body conditions described above, by kines- thetic matching of a 0 -deg rod. The hypothesis was that matching errors would increase in the supine position in the direction opposite to the tilted frame, consistent with increased rod-adjustment errors in the direction of frame tilt.

When the 0-deg rod is viewed in the presence of the 22.5-deg frame, both torsion and contrast effects may contribute to the illusion of rod tilt. If the rod and frame are rigidly rotated to certain other positions, however, only the contrast component should affect perceived rod orientation. For example, if the rod is tilted by $22.5 \mathrm{deg}$ and the frame is set at $45 \mathrm{deg}$, the angles between the rod and frame sides remain unchanged. If an illusory increase occurs in the angle between the rod and the sides of the frame most nearly parallel to the rod, then the rod should look less tilted than $22.5 \mathrm{deg}$. Since the frame is symmetrically oriented with respect to the gravitational and retinal axes, no frame-induced torsion should occur, however. The kinesthetic matching method is particularly useful for studying frame effects under these display conditions, since changes in the apparent orientation of oblique rods can be measured, as well as 0 -deg rods. The predicted kinesthetic-matching errors have been found when contrast effects are studied in this way (Goodenough, Oltman et al., 1979). The effect of a 45-deg frame on kinesthetic matches to 22.5-deg rods was examined in the present study to test the hypothesis that the contrast effect increases in the supine position.

Eye torsion, but no contrast effects, should occur under certain other display conditions. For example, if the rod is tilted by $22.5 \mathrm{deg}$, the addition of the frame at the same orientation as the rod should produce no contrast effect, but the rod should look less tilted due to visually induced eye torsion in the direction of display tilt. Under these display conditions, kinesthetic-matching errors have been found in erect observers in the predicted direction and on the same order of magnitude as objectively measured visually induced eye torsion (Goodenough, Oltman et al., 1979). They were therefore included in the present study to further examine the effect of body position on torsional responses to the tilted frame.

\section{Method}

The subjects were each given 22 kinesthetic-matching trials, followed by six egocentric rod-adjustment trials in the erect and in the supine positions. The sequence of body positions was erectsupine for a randomly selected half of the subjects and supineerect for the other half.

The rod-adjustment trials in each body position consisted of all combinations of two starting positions of the rod $(22.5 \mathrm{deg}$ $\mathrm{CW}$ and $\mathrm{CCW}$ ) and three frame conditions (absent, $22.5 \mathrm{deg} \mathrm{CW}$ and $\mathrm{CCW}$ ). The six trials were divided into two successive blocks of three trials, with each frame condition occurring once in a randomly chosen sequence within each block. The two starting positions for each frame and body condition were also assigned randomly to blocks.

The kinesthetic-matching method was adapted from Templeton (1972). In this method, the subject grasps handles that are mounted 
on opposite sides of a rotatable disk. The RF display is viewed through the hollow center of the disk and is the only visible stimulus. The subject's task is to align the disk handles with the rod (i.e., to rotate the handles until it seems to her as if she would be holding onto the ends of the rod if it were extended in both directions).

The kinesthetic-matching trials in each body position consisted of all combinations of two starting positions of the kinesthetic handles (45 deg CW and $\mathrm{CCW}$ ), and 11 rod-and-frame conditions as follows: frame absent with rod at $0 \mathrm{deg}, 22.5 \mathrm{deg} \mathrm{CW}$, and $22.5 \mathrm{deg} C \mathrm{CW}$; rod at $0 \mathrm{deg}$ with frame at $0 \mathrm{deg}, 22.5 \mathrm{deg} \mathrm{CW}$, and $22.5 \mathrm{deg} \mathrm{CCW}$; both rod and frame at $22.5 \mathrm{deg} \mathrm{CW}$ and $\mathrm{CCW}$; and frame at $45 \mathrm{deg}$ with rod at $0 \mathrm{deg}, 22.5 \mathrm{deg} C W$, and $22.5 \mathrm{deg}$ $\mathrm{CCW}$. The 22 trials at each body position were divided into two blocks of 11 trials, with each rod-and-frame condition occurring once within each block. The sequence of kinesthetic-matching trials was randomized in the same way as for the rod-adjustment trials described above.

Apparatus. The equipment included a padded table, a chair, and support structures to which two identical RF displays and kinesthetic disks were attached. Adjustable head clamps were mounted on the table and the back of the chair for maintaining the subject's head in a fixed position. The chair was provided with seat boards of varying thicknesses, which could be inserted, depending on the subject's sitting height, to adjust eye level to alignment with the rod and frame.

Each display was constructed of electroluminescent strips, with the rod and frame rotatable around a common central axis. The frame sides subtended $28 \mathrm{deg}$ of visual angle, as in the standard RFT. The disks had inside diameters of $45 \mathrm{~cm}$, with handles mounted on opposite sides so that an imaginary line could be drawn through the long axis of each handle and the center of disk rotation. In the erect position, the rod and frame were located $112 \mathrm{~cm}$ and the disk $26 \mathrm{~cm}$ in front of the subject's head. The centers of their rotation were located on a horizontal axis that intersected the subject's mid-eye position. In the supine position, rod, frame, and disk were located at the same distances above the head.

Procedure. The experimental trials were preceded by a 5 -min dark-adaptation period during which the subject was given practice trials at kinesthetically matching the visual rod alone. At the start of each trial, the subject's eyes were closed, her hands were in her lap (in the erect condition) or on her abdomen (in the supine condition), the rod-and-frame display was turned off, and the kinesthetic disk was placed in the starting position. The subject was first told which disk handle was "up" (right or left) for that trial and was asked to grasp the handles. The visual display was then turned on, and the subject opened her eyes and rotated the handles to match the rod. The display was then turned of $f$, and the subject returned her hands to her lap or abdomen and closed her eyes. There were approximately $30 \mathrm{sec}$ between trials. The rod-adjustment trials were administered immediately after the kinesthetic-matching trials in the same body position, following standard RFT procedures (e:g., Witkin et al., 1962/1974). Procedures used in the second body position were the same as those used in the first, except that the practice trials were omitted.

\section{Results}

Frame effects on egocentric rod adjustments and kinesthetic matches to the 0 -deg rod. Table 1 summarizes the data on the RF illusion, as measured by the egocentric rod-adjustment and kinesthetic-matching methods. As may be seen in the table, rod adjustments err in the direction of frame tilt, and kinesthetic matches err in the opposite direction, as found in most previous studies. The rod-adjustment errors increase in the supine position, as expected, but, unexpectedly, the kinesthetic-matching errors do not.
Table 1

Mean Rod-Adjustment and Kinesthetic-Matching Measures of the RF Illusion in Experiment 1

\begin{tabular}{lccr}
\hline & \multicolumn{3}{c}{ Frame Condition } \\
\cline { 3 - 4 } Position & $-22.5 \mathrm{deg}$ & Absent & $+22.5 \mathrm{deg}$ \\
\hline & \multicolumn{3}{c}{ Rod Adjustment } \\
Erect & -6.1 & -.3 & +6.2 \\
Supine & -8.3 & +2.1 & +10.0 \\
& Kinesthetic Matches with the 0-deg Rod \\
Erect & +3.0 & -.1 & -1.8 \\
Supine & +3.3 & -.1 & -1.4 \\
\hline
\end{tabular}

Note-Values are given in degrees.

Pooling the data from the CW and CCW frame conditions, the mean rod-adjustment error in the direction of frame tilt was $6.2 \mathrm{deg}$ for the erect position and $9.1 \mathrm{deg}$ for the supine position. The mean kinesthetic-matching error was $2.4 \mathrm{deg}$ in the opposite direction for both body positions. ${ }^{1}$

Measuring the effects of the tilted frame in terms of signed deviations from the rod-alone settings in the direction of frame tilt, analyses of variance show a significant main effect of frame orientation for rod adjustments $[F(1,18)=60.3, p<.01]$ and for kinesthetic matches $[F(1,18)=36.5, p<.01]$. Deviations in rod settings also show a significant interaction between frame condition and body position $[F(1,18)=$ $7.1, \mathrm{p}<.05]$, reflecting the erect-supine differences in the RF illusion when measured by the rodadjustment method. This interaction was insignificant in the kinesthetic-matching data, however.

Kinesthetic matches to the 22.5-deg rod. The data on kinesthetic matches to the tilted rod are summarized in Table 2.

Kinesthetic settings to the 22.5-deg rod in the absence of the frame tended to err systematically in the less tilted direction $[F(1,18)=25.7, p<.01]$. This result is consistent with previous findings that the most tilted rods in the set that the observer is asked to match tend to be underestimated (Gogel \& Newton, 1975; Goodenough, Oltman et al., 1979). The ten-

Table 2

Mean Kinesthetic Settings as a Function of Rod, Frame, and Body Orientations in Experiment 1

\begin{tabular}{lccc}
\hline \multirow{1}{*}{$\begin{array}{c}\text { Frame } \\
\text { Condition }\end{array}$} & \multicolumn{3}{c}{ Rod Orientation } \\
\cline { 2 - 4 } & $-22.5 \mathrm{Deg}$ & 0 Deg & $+22.5 \mathrm{Deg}$ \\
\hline Absent & -19.8 & -.1 & +22.0 \\
45 Deg & -17.3 & -.4 & +19.2 \\
Aligned With Rod & -18.8 & -.9 & +21.2 \\
& & Supine & \\
Absent & -17.6 & -.1 & +20.3 \\
45 Deg & -16.8 & -.4 & +18.4 \\
Aligned With Rod & -18.0 & +.3 & +20.2 \\
\hline
\end{tabular}

Note-Values are given in degrees. 
dency to underestimate rod tilt was greater for the CCW rod than for the $C W \operatorname{rod}[F(1,18)=7.6$, $\mathrm{p}<.05]$ and was greater for the supine than for the erect body position $[F(1,18)=15.8, p<.01]$.

The tendency to underestimate the 22.5-deg rod was significantly greater in the presence of the 45-deg frame than in its absence $[F(1,18)=40.5, p<.01]$. This frame effect is similar to previous findings in the erect position (Goodenough, Oltman et al., 1979) and is consistent with an orientation contrast effect. With respect to body position, no significant erectsupine difference was found in the effect of the 45-deg frame. Thus, no evidence was found in these data that the orientation contrast component increases in the supine position.

In the erect position, the tendency to underestimate the tilt of the 22.5-deg rod was also significantly greater with the 22.5-deg frame present than with the rod alone $[F(1,18)=6.3, p<.05]$. This result is similar to previous findings and is consistent with a visually induced torsional response in the direction of frame tilt (Goodenough, Sigman et al., 1979). A significant interaction $[F(1,18)=6.3, p<.05]$ was also found between the effect of frame condition (present vs. absent) and body position. The interaction is due to the fact that, unlike the erect position, the addition of the frame at the same orientation as the rod had no significant effect in the supine position. The pattern of results is difficult to interpret when the frame is aligned with the rod, but there is certainly no support in these data for the view that torsion increases in the supine position.

\section{Discussion}

The data from the first experiment clearly confirm previous findings that the effect of a tilted frame on egocentric rod adjustments increases in the supine position, but the finding that a similar increase does not occur in the kinesthetic-matching data under any display condition suggests that the erect-supine difference is not due to either a torsion or contrast component of the RF illusion.

\section{EXPERIMENT 2}

The hypothesis that visually induced eye torsion increases in the supine position was examined in this experiment by objective measurements of eye orientation.

\section{Method}

Torsional responses were measured photographically in eight new subjects while monocularly viewing the 22.5 -deg $\mathrm{CW}, 0$-deg, or 22.5-deg CCW frame with the right eye. There were 15 trials for each subject in the erect and supine positions, divided into five successive blocks of three trials. Each of the three display conditions occurred once in a randomly determined sequence within each block. Half of the subjects were run in the supine position first, and the remainder were run in the erect position first.

Apparatus. A Nikkormat Model FT2 camera was mounted so that its optical axis was oriented at $90 \mathrm{deg}$ to the line of sight between the right eye and the fixation point. A half-silvered mirror was mounted at a 45-deg angle to both the line of sight and the camera axis, so that the subjects viewed the display through the mirror and the image of the eye was reflected to the camera.

Procedure. During each trial, the subject was asked to look at the frame for $15 \mathrm{sec}$ and to fixate a center light during the last $3 \mathrm{sec}$ of this period, at which time the photograph was taken. There was a 30 -sec rest period in the dark between trials.

The photographic method was adapted from Howard and Evans (1963) and is described in detail elsewhere (Goodenough, Sigman et al., 1979). The display was viewed through the center of a ring that was attached to a bite plate worn by the subjects. Two landmarks were etched on the ring and were visible in the photographs for use in measuring head orientation. Two scleral landmarks were used to measure eye orientation with respect to the ring landmarks. Measurements of eye orientation relative to the head were made by projecting the photographs onto a paper with a magnification factor of 26. Using this technique, the standard error of measurement was found to be $.1 \mathrm{deg}$ for data collected on an artificial eye (Goodenough, Sigman et al., 1979).

Angle differences between the frame-upright and frame-tilted conditions were assigned a plus value if in the direction of frame tilt and a minus value if in the opposite direction. The mean of these algebraic differences was then used to assess frame effects on eye orientation.

\section{Results and Discussion}

No significant differences in amount of eye torsion were found as a function of direction of display tilt. $\mathrm{CW}$ and $\mathrm{CCW}$ trials were therefore pooled in subsequent data analyses. Significant eye torsion in the direction of display tilt was found in both the erect $[F(1,7)=14.00, p<.01]$ and supine $[F(1,7)=12.74$, $\mathrm{p}<.01]$ positions. The mean torsional response was slightly greater in the supine $(.59 \mathrm{deg})$ than in the erect $(.45 \mathrm{deg})$ recordings, but this difference is not significant.

The estimates of torsional amounts differ somewhat between the photographic method of Experiment 2 and the kinesthetic-matching method of the first experiment. In general terms, however, the data from both studies suggest that eye torsion is not a very important factor in the RF illusion under any of the conditions examined and are consistent with the conclusion of Finke and Held (1978) that visually induced torsion does not increase very much, if at all, in the supine position.

\section{EXPERIMENT 3}

The failure in the first experiment to find any erectsupine difference in the effect of the 22.5-deg frame on disk adjustments to the $0-\mathrm{deg}$ rod is particularly interesting because it supports the view that kinestheticmatching and rod-adjustment methods are not interchangeable measures of the RF illusion. It seems reasonable to assume that both methods would be affected by torsion and contrast components. The find- 
ing that erect-supine differences occur only for rodadjustment errors suggests, therefore, that some additional, unique component of the rod-adjustment task is responsible for the effect of body position.

One obvious difference between the rod-adjustment and kinesthetic-matching tasks is that the observer must imagine a line running through the eyes in the former, but this line is supplied by the rod in the latter. Thus, accurate judgments about which egocentric orientation corresponds to the head axis are required only by the rod-adjustment method. If the observer's internal representation of egocentric orientation is shifted in the direction of frame tilt, then kinesthetic matches to the visible rod should not be affected by the frame, but either rod or disk adjustments to the body axis should err in the direction of frame tilt. This possibility was examined in the third experiment.

\section{Method}

Erect-supine differences in the effects of the 22.5-deg frame were examined in this experiment under two kinesthetic-adjustment conditions. The first was the same as the kinesthetic-matching condition of Experiment 1 with the 0 -deg rod and 22.5-deg frame. The second was the same as the rod-adjustment condition of Experiment 1 except that the rod was turned off and the subjects were instructed to adjust the disk handles (instead of the rod) to an imaginary line running through the centers of both eyes. If the requirement for accurately representing the body axis is a necessary and sufficient condition for obtaining erect-supine differences in the frame effects, then these differences should occur with egocentric kinesthetic adjustments, as with egocentric rod adjustments, but not with kinesthetic matches to the visible rod.

Forty-eight trials were run on each of eight additional female subjects. They consisted of two trials under each combination of the two body positions (erect, supine), two rod conditions (present, absent), three frame orientations $(0 \mathrm{deg}, 22.5 \mathrm{deg} \mathrm{CW}$, and $22.5 \mathrm{deg}(\mathrm{CW})$, and two starting positions of the disk (45 deg $\mathrm{CW}$ and $\mathrm{CCW}$ ). The equipment and procedures were otherwise the same as in Experiment 1.

\section{Results}

The data of Experiment 3 are summarized in Table 3. As in Experiment 1, no significant erectsupine difference was found in kinesthetic matches to the 0-deg rod. The mean signed error was opposite in direction from the frame tilt for both the erect $(2.8 \mathrm{deg})$ and supine $(3.1 \mathrm{deg})$ body positions. A sig-

Table 3

Mean Kinesthetic Settings in Experiment 3

\begin{tabular}{lccc}
\hline & \multicolumn{3}{c}{ Frame Condition } \\
\cline { 2 - 4 } Position & $-22.5 \mathrm{Deg}$ & 0 Deg & $+22.5 \mathrm{Deg}$ \\
\hline & \multicolumn{3}{c}{ 0-Deg Rod Condition } \\
Erect & +3.7 & +.7 & -1.9 \\
Supine & +3.2 & +.2 & -3.0 \\
& \multicolumn{4}{c}{ Absent Rod Condition } \\
Erect & -.1 & +.6 & +1.0 \\
Supine & -2.9 & +.5 & +3.0 \\
\hline
\end{tabular}

Note-Values are given in degrees. nificant erect-supine difference was found, however, in kinesthetic adjustments to the head axis $[F(1,7)=$ $7.1, \mathrm{p}<.05]$. The mean disk settings, signed in the direction of frame tilt, were .5 deg in the erect position (not significantly different from zero) and $3.0 \mathrm{deg}$ in the supine position $[\mathrm{F}(1,7)=38.4, \mathrm{p}<.01]$.

\section{Discussion}

The results of the third experiment are consistent with the view that a shift toward the tilted frame occurs in the observer's internal representation of egocentric space, at least in the supine position.

An error component with similar properties has been identified in studies on judging the egocentric orientation of a stimulus with respect to the median plane (e.g., Glick, 1966; Harris, 1974). ${ }^{2}$ Harris, for example, has suggested that there are two different frames of reference that observers may use to define the straight-ahead direction. One is the strictly egocentric reference in which straight-ahead is defined by the observer's median plane, and the other is an environmental standard in which the straight-ahead is defined by the major axis of the external world (e.g., the room or a laterally displaced frame). While subjects' judgments may be influenced by instructional variations in the emphasis placed on egocentric or environmental standards (e.g., Glick, 1966), the observer may have some difficulty in completely separating these two standards (e.g., Harris, 1974). Thus, compromise judgments, called "straightahead shifts" by Harris, might be expected.

A distinguishing property of the straight-ahead shift is its modality nonspecificity, in the sense that errors are expected whether the subject is asked to point in the straight-ahead direction or to adjust a point of light to the straight-ahead, but errors in pointing to a spot of light are not expected. The component of the egocentric RF task that appears responsible for erect-supine differences in the present studies is distinguishable in terms of similar properties. Thus, an "orientation shift" may occur in the direction of the environmental reference defined by the tilted frame, resulting in compromise judgments about the orientation of the body axis as measured by egocentric settings of either rod or disk, but not affecting kinesthetic matches to the visible rod.

It seems reasonable to suppose that the environmental axes are jointly determined by visual and gravitational cues in the erect position. If this view is correct, then egocentric RF errors may be lower in the erect position due to a dilution by gravitational cues of the role of the tilted frame in defining the environmental axes. Thus, environmental and egocentric standards for judging orientation may differ less in the erect, than in the supine, position, and confusion between the two standards may in turn result in smaller egocentric errors in the erect position. 


\section{REFERENCES}

Brosgole, L., \& Cristal, R. M. The role of phenomenal displacement on the perception of the visual upright. Perception \& Psychophysics, 1967, 2, 179-188.

Carpenter, R. H. S., \& Blakemore, C. Interactions between orientations in human vision. Brain Research, 1973, 18, 287-303.

Crone, R. A. Optically induced eye torsion II: Optostatic and optokinetic cycloversion. Albrecht von Graefes Archiv Klinische und Experimentelle Ophthalmologie, 1975, 196, 1-7.

Finke, R. A., \& HELD, R. State reversals of optically induced tilt and torsional eye movements. Perception \& Psychophysics, $1978,23,337-340$.

GLick, J. An experimental analysis of subject-object relationships in perception. In S. Wapner \& B. Kaplan (Eds.), Essays in honor of Heinz Werner. Worcester, Mass: Clark University Press, 1966.

Gogel, W. C., \& Newton, R. E. Depth adjacency and the rodand-frame illusion. Perception \& Psychophysics, 1975, 18, 163-171.

Goodenough, D. R., Oltman, P. K., Sigman, E., Rosso, J., \& MERTZ, H. Orientation contrast effects in the rod-and-frame test. Perception \& Psychophysics, 1979, 25, 419-424.

Goodenough, D. R., Sigman, E., Oltman, P. K., Rosso, J., \& Mertz, H. Eye torsion in response to a tilted visual stimulus. Vision Research, 1979, 19, 1177-1179.

HARRIS, C. W. Beware of the straight-ahead shift-A nonperceptual change in experiments on adaptation to displaced vision. Perception, 1974, 3, 461-476.

Howard, I. P., \& Evans, J. A. The measurement of eye torsion. Vision Research, 1963, 3, 447-455.

Hughes, P. C. The influence of the visual field upon the visual vertical in relation to ocular torsion of the eye (Doctoral dis- sertation, University of Oklahoma, 1973). Dissertation Abstracts International, 1973, 33, 4686B. (University Microfilms No. 739158)

Lichtenste in, J. H., \& Saucer, R. T. Visual dependency in the erect and supine positions. Journal of Applied Psychology, 1974, 59, 529-531.

Sigman, E., Goodenough, D. R., \& Flannagan, M. Instructions, illusory self-tilt, and the rod-and-frame test. Quarterly Journal of Experimental Psychology, 1979, 31, 155-165.

Templeton, W. B. Visual normalization: The method of kinesthetic matching. Perception \& Psychophysics, 1972, 12, 422-424.

Templeton, W. B. The role of gravitational cues in the judgment of visual orientation. Perception \& Psychophysics, 1973, 14, 451-457.

Wallace, G. K., \& Moulden, B. The effect of body tilt on the Zöllner illusion. Quarterly Journal of Experimental Psychology, $1973,25,10-21$.

Witkin, H. A., Dyk, R. B., Faterson, H. F., Goodenough, D. R., \& KArP, S. A. Psychological differentiation. Potomac, Md: Erlbaum, 1974. (Originally published by Wiley, 1962.)

\section{NOTES}

1. As the tabled means suggest, the $\mathrm{CCW}$ frame had a larger effect on kinesthetic matches than did the $C W$ frame $[F(1,18)=11.2$, $\mathrm{p}<.01]$.

2. We are indebted to Irvin Rock for calling our attention to this similarity.

(Received for publication July 21, 1980; accepted January 19,1981 .) 